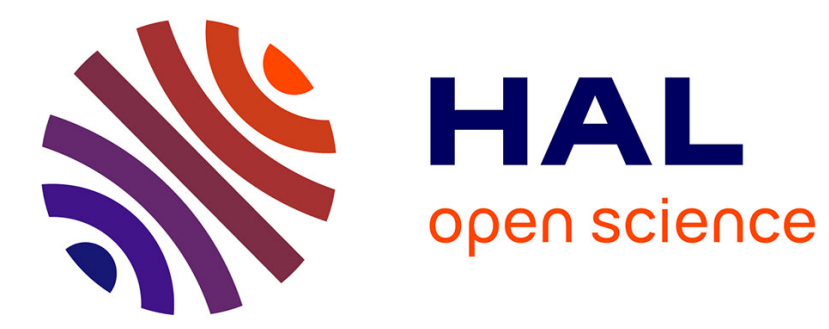

\title{
ON THRESHOLDS FOR SURFACE WAVES ON RESONANT BUBBLES
}

\author{
R. Nabergoj, A. Francescutto
}

\section{To cite this version:}

R. Nabergoj, A. Francescutto. ON THRESHOLDS FOR SURFACE WAVES ON RESONANT BUBBLES. Journal de Physique Colloques, 1979, 40 (C8), pp.C8-306-C8-309. 10.1051/jphyscol:1979854 . jpa-00219560

\section{HAL Id: jpa-00219560 https://hal.science/jpa-00219560}

Submitted on 1 Jan 1979

HAL is a multi-disciplinary open access archive for the deposit and dissemination of scientific research documents, whether they are published or not. The documents may come from teaching and research institutions in France or abroad, or from public or private research centers.
L'archive ouverte pluridisciplinaire HAL, est destinée au dépôt et à la diffusion de documents scientifiques de niveau recherche, publiés ou non, émanant des établissements d'enseignement et de recherche français ou étrangers, des laboratoires publics ou privés. 
ON THRESHOLDS FOR SURFACE WAVES ON RESONANT BUBBLES

\author{
NABERGOJ R., and FRANCESCUTTO A. \\ Istituto di Fisica delZ'Università di Trieste, 34127 - Trieste, Italy
}

Résumé. - Le problème de la stabilitê de bulles gazeuses dans un champ sonore a été étudiē à l'aide d'un modèle décrivant le mouvement d'une bulle presque sphérique dans un liquide légèrement visqueux. L'amplitude du mouvement radial et les seuils d'amplitude de pression pour l'instabilité d'une forme sphërique ont été obtenu au moyen d'une théorie de résonance paramétrique. La comparaison avec les résultats expérimentaux pour les bulles en résonance apparait comme assez satisfaisante.

Abstract. - The problem of the stability of gas bubbles in a sound field has been investigated using a model for the motion of a nearly spherical bubble in a slightly viscous liquid. The radial motion amplitude and the pressure amplitude thresholds for the instability of the spherical shape have been obtained by the theory of parametric resonance. The comparison with available experimental data for resonant bubbles appears to be rather satisfactory.

1. Introduction. - The mechanism of acoustic cavitation is closely influenced by the behavior of gas bubbles in the liquid. In real situations, a pulsating bubble does not maintain the spherical shape, due to the onset of surface waves at a particular threshold. When the amplitude of the deformations is sufficiently high, microbubbles can be generated at the bubble surface /1/. It appears that certain combinations: of bubble radius and drinving frequency are especially effective in bubble formation. This fact is related to the parametric excitation of surface waves by radial oscillations.

The aim of the present work is to obtain the threshold in terms of the amplitude of the radial motion or in terms of the corresponding pressure amplitude of the driving fields. The viscous effects are directly introduced in the equations of motion for the non-spherical bubble, and this represents the main difference from the preceding theories of Hsieh-Plesset /2,3/ and Benjamin /4,5/.

The general theory of the parametric excitation of surface waves on pulsating gas bubbles is considered in Part 2. Simplified formulas of the thresholds for resonant bubbles are given in Part 3.

2. Parametric excitation of surface waves. - Let us consider a nearly spherical buible in an incompressible, unbounded liquid of small viscosity. As usual $16,7 /$, the free surface may be described in terms of spherical harmonics $Y_{n}$, that is

$r_{s}(V, t)=R(t)+\Sigma_{n} a_{n}(t) Y_{n}(\mathscr{q})$, where $a_{n}(t)$ are the amplitudes of the surface deformations and $R(t)$ is the mean radius, obtained from the Rayleigh-Plesset equation. The behavior of the surface for $\left|a_{n}\right|<<$ can be obtained in the limit of small amplitude oscillations, and in the linearized theory, the equations describing the different amplitudes become uncoupled. Insofar as smalT viscosity liquids are considered, elsewhere /8/ we obtained, by using the Lagrangian procedure with a dissipation function, the equation for the $a_{n}$ 's in the form

$$
\begin{aligned}
\ddot{a}_{n} & +\left[3 \dot{R} / R+2(n+2)(2 n+1) \mu / \rho R^{2}\right] \dot{a}_{n}+\left[(n-1)(n+1)(n+2) \sigma / \rho R^{3}\right. \\
& \left.-(n-1) \ddot{R} / R+2(n-1)(n+2) \mu \dot{R} / \rho R^{3}\right] a_{n}=0
\end{aligned}
$$

where $\rho$ is the density of the riquid, $\sigma$ the surface tension and $\mu$ the viscosity. In the exact solution the equation of motion is more involved $/ 9 /$, having an integro-differential structure, except in the case of negligible boundary layer effects, where Eq. 1 is recovered /10/.

By constant radius $R(t)=R_{0}$, that is for surface oscillations about the equilibrium position, Eq. 1 reduces to the equation for a damped harmonic oscillator of natural frequency

$$
\begin{aligned}
& \omega^{2} . \text { on }=(n-1)(n+1)(n+2) \sigma / \rho R_{0}^{3}, \\
& \text { and damping constant } \\
& \beta_{n}=(n+2)(2 n+1) \mu / \rho R_{0}^{2} .
\end{aligned}
$$


In the study of the surface distortions it is expedient to set

$a_{n}=b_{n}\left(R_{j} / R\right)^{3 / 2} \exp \left[-(n+2)(2 n+1) \mu / \rho \int_{0}^{t} d t^{\prime} / R^{2}\right]$,

where $R_{i}=R(0)$, so that Eq.1 takes the form

$\ddot{b}_{n}+G(t) b_{n}=0$,

with

$$
\begin{aligned}
G(t) & =(n-1)(n+1)(n+2) \sigma / \rho R^{3}-\frac{3}{4}(\dot{R} / R)^{2}-\left(n+\frac{1}{2}\right) \ddot{R} / R \\
& -3(n+2) \mu \dot{R} / \rho R^{3}-\left[(n+2)(2 n+1) \mu / \rho R^{2}\right]^{2} .
\end{aligned}
$$

For understanding surface phenomena it is important to consider the mechanism of excitation of surface deformations. Clearly, the results of the linearized approximation cannot be valid for surface oscillations of high amplitude when the breakup of the bubble takes place. However, for oscillations of small amplitude, Eq.1 is adequate for describing several features of the motion and in particular the development of an instability of the spherical shape. The surface distortions of a vibrating bubble can, in particular, be parametrically excited by the radial pulsations.

For considering this we assume that, due to a superimposed acoustic field, the external pressure oscillates about its average static value $p_{\infty}$ with angular frequency $\omega$, that is

$p(t)=p_{\infty}(1+n \cos \omega t)$.

When the acoustic field is sufficiently weak, that is $n \ll 1$, the radial oscillation will be approximately simple harmonic. A linearized computation on Rayleigh-Plesset equation then gives

$$
R(t)=R_{0}[1+c \cos (\omega t+\phi)] \text {, }
$$

where the relative pulsation amplitude $C=\Delta R / R_{0}$ is of the same order of magnitude of $n$, and $\phi$ is a constant phase shift which for convenience may be put equal to zero. By substituting Eq.3 and retaining only linear terms in C, Eq.1 takes the form of a Mathieu equation,

$\ddot{\mathrm{b}}_{\mathrm{n}}+\left[\omega_{n}^{2}+\alpha_{n} \cos \left(\omega t-\varepsilon_{n}\right)\right] \mathrm{b}_{n}=0$,

where

$\omega_{n}^{2}=\omega_{o n}^{2}-\beta_{n}^{2}, \alpha_{n}=C\left\{\left[-3 \omega_{o n}^{2}+\left(n+\frac{1}{2}\right) \omega^{2}+4 \beta_{n}^{2}\right]^{2}\right.$

$\left.+x_{n}^{2} \omega^{2}\right\} 1 / 2, \varepsilon_{n}=\operatorname{arctg}\left\{x_{n} \omega /\left[-3 \omega_{o n}^{2}+\left(n+\frac{1}{2}\right) \omega^{2}+4 \beta_{n}^{2}\right]\right\}$, and $x_{n}=3(n+2) \mu / \rho R_{0}^{2}$. It is well known from the theory of the Mathieu equation that, for particular combinations of the constants $\alpha_{n}$ and $\omega_{n}^{2}$, the solutions may have the form of modulated oscillations with amplitude exponentially growing with time. This behavior indicates that parametric excitation of surface waves is possible, giving rise to the instability of the spherical shape.

Limiting our analysis to the first instability zone, a particulai solution of Eq. 4 is given /11/ by

$b_{n}(t)=\phi_{n}(t) \exp \left(x_{n} t\right)$

with

$x_{n}^{2}=-\left(\omega_{n}^{2}+\frac{1}{4} \omega^{2}\right)+\left(\omega_{n}^{2} \omega^{2}+\frac{1}{4} \alpha_{n}^{2}\right)^{1 / 2}$,

and $\phi_{n}(t)$ a periodic function subresonant $1 / 2$ to the frequency of the forcing field. We have

$a_{n}(t)=\phi_{n}(t) \quad \exp \left[\left(x_{n}-\beta_{n}\right) t\right]$,

that is the amplification of the surface oscillations due to parametric resonance follows the law $\exp \left(x_{n}-\beta_{n}\right) t$, the boundary of the instability zone being given by $x_{n}-\beta_{n}=0$. After straight forward calculations, the pulsation amplitude threshold for the onset of surface waves is then obtained in the form

$$
\begin{aligned}
c_{t} & =2\left[\left(\omega_{o n}^{2}-\frac{1}{4}{ }^{2}\right)^{2}+\beta_{n}^{2} \omega_{n}^{2}\right]^{1 / 2}\left[\left[-3 \omega_{o n}^{2}+\left(n+\frac{1}{2}\right) \omega^{2}+4 \beta_{n}{ }^{2}\right]^{2}\right. \\
& \left.+x_{n}^{2}{ }^{2}{ }^{2}\right]^{-1 / 2}
\end{aligned}
$$


In the small viscosity approximation $\beta_{n}<<\omega_{\text {on }}$ and $x_{n}<\omega_{o n}$, so that a simplified expression for $c_{t}$ can be obtained. For the subresonance region, where $\omega \simeq 2 \omega_{\text {on }}$, by retaining the leading terms, it results

$c_{t} \simeq 2(4 n-1)^{-1}\left[\left(1-\omega^{2} / 4 \omega_{o n}^{2}\right)^{2}+4 \beta_{n}^{2} / \omega_{o n}^{2}\right]^{1 / 2}:$

For the linear bubble oscillations, the pressure amplitude can be easily expressed in terms of the pulsation amplitude. The relative pressure amplitude threshold is then given by

$\eta_{t}=\left(\rho R_{0}^{2} c_{t} / p_{\infty}\right)\left[\left(\omega_{0}^{2}-\omega^{2}\right)^{2}+4 \beta_{e q} q^{2}\right]^{-1 / 2}$,

where

$\omega_{0}=\left(3 k p_{\infty} / \rho R_{0}^{2}\right)\left[1+2 \sigma(3 k-1) / 3 k p_{\infty} R_{0}\right]^{1 / 2}$ is the bubble resonance frequency for linear oscillations, $k$ the polytropic exponent of the gas transformation, and $\beta_{\mathrm{eq}}=2 \mu_{\mathrm{eq}} / \rho \mathrm{R}_{0}^{2}$, with $\mu_{\mathrm{eq}}$ the effective viscosity of the liquid taking into account the viscous, thermal and radiation effects.

3. Discussion and conclusions. - Fig. 1. shows the curves $C_{\text {to }}=C_{t}\left(\omega_{0}\right)$ versus the rest radius $R_{0}$

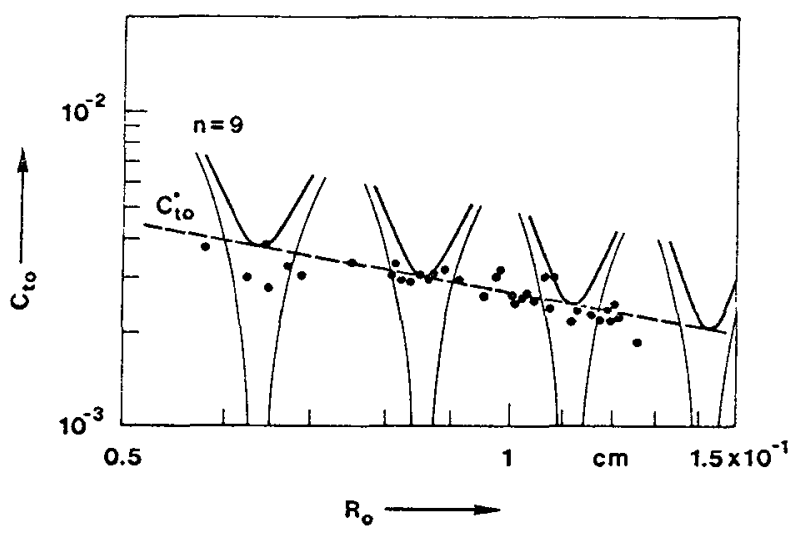

Fig. 1. - Predicted relative pulsation amplitude threshold $C_{\text {to }}=\Delta R / R$ versus rest radius $R$ for bubbles in water driven at resonance, together with the "effective" threshold $C_{\text {to. }}$. The thin line curves represent the threshold according to Hsieh-Plesset for zero viscosity. The experimental points, relative to standing tap water, are taken from Fig. 6b of Ref. 12. in the range $5 \times 10^{-2} \mathrm{~cm} \leqslant R_{0} \leqslant 1.5 \times 10^{-1} \mathrm{~cm}$ for bubbles in water driven at resonance. For comparison we show also the experimental data of Hullin 112/. The agreement between theory and experiment is quite satisfactory.

The dashed line joining the minima could be assumed as the "effective" threshold, the instability region covers the upper part of the plane. This threshold $C_{\text {to }}$ is suggested by the fact that in the region between the minima of subharmonic resonance there will presumably appear other minima, corresponding to zones of instability, which can not be taken into account in this first order analysis. A simple approximate formula is readily obtained from Eq. 5 in the limit of large values of $n$, namely

$$
C_{\text {to }}^{*} \text { A } A \mu \rho-1 / 2\left(\sigma R_{0}^{2}\right)^{-1 / 3} \text {, }
$$

with $A \simeq 0.25$ c.g.s. units.

In Fig. 1 we also give a comparison of the present theory with that given by Hsieh-Plesset $12,3 /$. It appears that the latter, based on zero damping assumption, does not allow an evaluation of an effective threshold.Moreover, in the considered range of radii the Benjamin's threshold $/ 5 /$, where the viscous effects are considered rather artificially "a posteriori" and not directly introduced by the equation of motion, is practically coincident with the present one.

Fig. 2 shows the pressure amplitude threshold $p_{\text {to }}=n_{t_{0}} p_{\infty}$ versus the rest radius $R_{0}$ relative to several surface modes, for bubbles in water driven at resonance. For this estimation the effective viscosity coefficient and the polytropic index were taken from Ref. 13. It appears that the "effective" pressure threshold for large radi $i$ is given by the approximate law

$$
\begin{aligned}
& p_{\text {to }}^{*} \simeq K R_{0}^{-1}, \\
& \text { where } K \simeq 50 \text { c.g.s. units. }
\end{aligned}
$$

A quantitative indication given by Storm /14/ for a bubble with $R_{0} \simeq 0.5 \mathrm{~cm}$ Ties one order of magnt tude above the value given by this formula. Many perturbing factors could account for this difference. For example, experiments /12/ in 


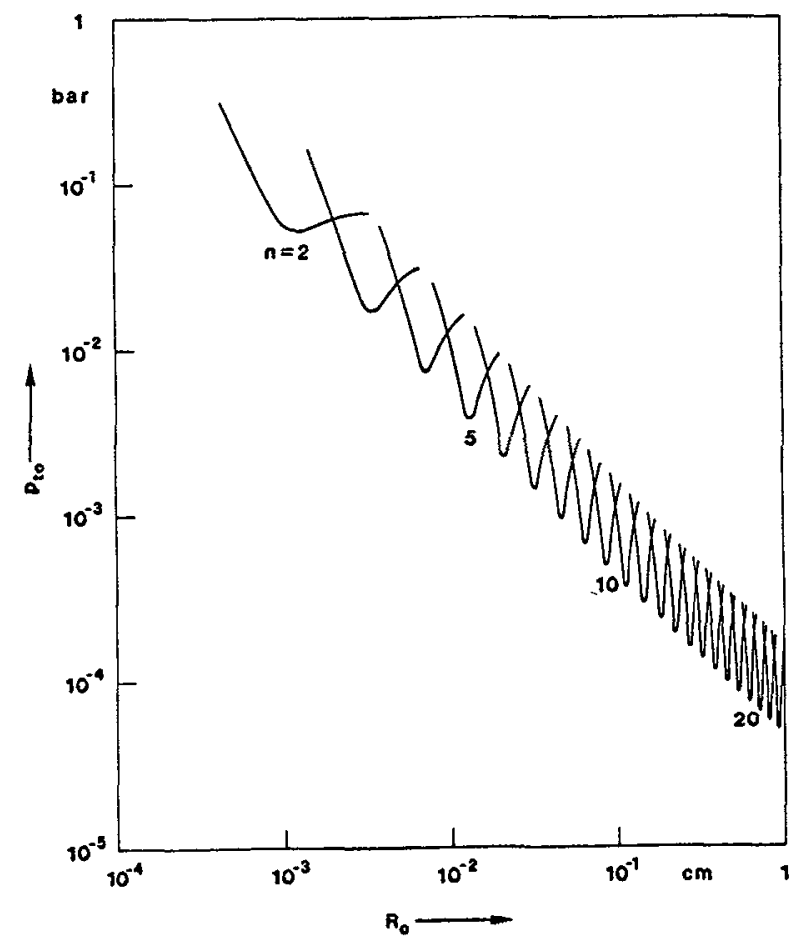

Fig. 2. - Predicted pressure amplitude threshold $p_{\text {to }}$ versus rest radius $R_{0}$ for bubbles in water driven at resonance. The number of each curve refers to the particular surface mode.

standing water show an increase of the threshold probably due to the impurities collected by the bubble surface. Further investigations could shed more light on this.

\section{REFERENCES}

/1/ Willard, G.W., J. Acoust, Soc. Am. 26 (1954) $933(A)$.

12/ Hsieh, D.Y., and Plesset, M.S., J. Acoust. Soc. Am. 33 (1961) 206.

/3/ Hsieh, D.Y., J. Acoust. Soc. Am. 56 (1974) 392.

14/ Benjamin, T.B., and Strasberg, M., J. Acoust. Soc. Am. 30 (1958) 697 (A).

15/ Benjamin, T.B., in Cavitation in Real Liquids (R. Davies ed., Elsevier Publishing Co., Amsterdam, 1964) pp. 164-180.

16/ Plesset, M.S., J. App 1. Phys. 25 (1954) 96.

17/ Hsieh, D.Y., J. Basic Eng. 94 (1972) 665.

18/ Ceschia, M., and Nabergoj, R., Phys. Fluids 21 (1978) 140?

19/ Prosperetti, A., Quart. App1. Math. 34 (1977) 339.

110/ Plesset, M.S., and Prosperetti, A., Ann. Rev. Fluid Mech. $\underline{9}$ (1977) 145.

/11/ Hayashi, C., Nonlinear Oscillations in Physical Systems (Mc Graw-Hill Co., New-York, 1964);

/12/ Hullin, Ch., Acustica 37 (1977) 64.

113/ Chapman, R.B., and Plesset, M.S., J. Basic Eng. 93 (1971) 373.

/14/ Storm, D.L., J. Acoust. Soc. Am. 52 (1972) $152(A)$. 